\title{
Financing Innovative Medicines in Mainland China: The Role of Commercial Health Insurance
}

\author{
Hong Li ${ }^{1,2,3}$, Gordon G. Liu ${ }^{4}$, Christoph Glaetzer ${ }^{5}$ \\ ${ }^{1}$ Bristol-Myers Squibb Company, Wallingford, USA \\ ${ }^{2}$ Cincinnati University, Cincinnati, USA \\ ${ }^{3}$ Shanghai Jiao Tong University, Shanghai, China \\ ${ }^{4}$ Peking University, Beijing, China \\ ${ }^{5}$ Janssen Pharmaceutical Companies of Johnson \& Johnson, Singapore City, Singapore \\ Email: hong.li@bms.com
}

Received June $1^{\text {st }}$, 2013; revised July $8^{\text {th }}$, 2013; accepted July $16^{\text {th }}, 2013$

\begin{abstract}
Copyright (C) 2013 Hong Li et al. This is an open access article distributed under the Creative Commons Attribution License, which permits unrestricted use, distribution, and reproduction in any medium, provided the original work is properly cited.
\end{abstract}

\begin{abstract}
The National Reimbursement Drug List (NRDL) is a key component of the current government-led insurance policy to provide access to basic medicines in mainland China; yet NRDL coverage is limited due to public budgetary constraints and new, innovative medicines are often excluded. This paper explores the potential role of commercial health insurance as an optional approach to financing medicines excluded by the NRDL. The current status of commercial health insurance (CHI) in Mainland China is reviewed and we discuss proposed changes that may be needed to develop an effective and sustainable commercial insurance program to provide the Chinese population with better access to innovative medicines not covered by NRDL.
\end{abstract}

Keywords: Mainland China; NRDL; Innovative Medicines; Commercial Health Insurance; Pharmaceutical

\section{Introduction}

Funding the increasing demands for access to health care is a constant topic for health policy globally. In Mainland China (hereafter referred to "China"), there are three primary government-run health insurance programs, including the Urban Employee Basic Medical Insurance (UEBMI), the Urban Resident Basic Medical Insurance (URBMI), and the New Rural Cooperative Medical Scheme (NRCMS) for the rural population (Hougaard et al., 2008; Sussmuth-Dyckerhoff \& Wang, 2010). With a strong commitment from the central government including budget allocation, the China Healthcare Reform Blueprint, published in April 2009 by the State Council, planned to expand the basic medical security system to provide coverage to all residents in both urban and rural areas (China healthcare reform, 2012). Since this blueprint was published, nearly $\$ 18.5$ billion USD has been allocated by the Chinese government at all levels to boost the government health insurance programs throughout the country. In November 2012, the China central government announced the allocation of an additional \$18.5 billion USD for fiscal year 2013 to continue expanding the government health insurance program (China government announcement, 2012). As a result of these efforts, government health insurance coverage in China reached 95\% in 2012, covering 1.27 billion people out of a 1.34 billion national population. The increased coverage by the government health insurance programs has been a strong reason to stimulate the national healthcare spending in China. It is expected that healthcare spending in China would be $\$ 1$ trillion USD in 2020, a substantial increase from \$357 billion USD in 2011 (Le Deu et al., 2012).

Currently in China, drug costs are relatively high compared to physician consultation fees, and hospital bed charges and total healthcare costs. This difference is mainly due to the cost structure in the current medical system in China. Published data from a survey of 21,520 hospitals across China in 2011 shows that drug costs were $50.6 \%$ and $41.8 \%$ of total medical costs in the outpatient setting and discharged admission, respectively (MOH China statistics, 2012). Recent reports on out-of-pocket expenses for healthcare spending in China have been reported to range as high as $60 \%$ in 2000 to $38.2 \%$ in 2009 (Table 1); this is relatively high compared to other countries (Table 2) (OECD, 2011; Bhattacharjye \& Sapra, 2008). In 2011, there were 6.3 billion outpatient visits and 153 million discharged hospitalizations across all medical facilities in China (MOH China Statistics, 2012). Assuming 30\% and 20\% coinsurance for medicines incurred by patients for outpatient and inpatient settings, respectively, thus the estimated out-of-pocket expenses for medicines in 2011 alone would be $\$ 269.7$ billion USD for outpatients and \$13.4 billion USD for inpatients in China.

Through the government-run health insurance systems, drugs on the formulary list can be reimbursed at various levels. The national formulary is updated every two years, starting with the central government, after which local governments are free to adapt and create their own local formularies. The most recent officially updated version (2009) of the NRDL included 1140 Western medicines (by chemical names) and 987 Chinese medi- 
LI H. ET AL.

Table 1.

Components of the total health expenditure in China (\%), from 1980 to 2010.

\begin{tabular}{cccccccccccc}
\hline Component/year & 1980 & 1990 & 1995 & 2000 & 2005 & 2006 & 2007 & 2008 & 2009 & 2010 \\
\hline From Government & 36.2 & 25.1 & 18.0 & 15.5 & 17.9 & 18.1 & 22.3 & 24.7 & 27.2 & 28.7 \\
Out of Pocket & 21.2 & 35.7 & 46.4 & 59.0 & 52.2 & 49.3 & 44.1 & 40.4 & 38.2 & 35.3 \\
All Others & 42.6 & 39.2 & 35.6 & 25.6 & 29.9 & 32.6 & 33.6 & 34.9 & 34.6 & 36.0 \\
Total & 100.0 & 100.0 & 100.0 & 100.0 & 100.0 & 100.0 & 100.0 & 100.0 & 100.0 & 100.0 \\
\hline
\end{tabular}

Table 2.

Out of pocket expenditure on health as a percentage of total expenditure on health from selected OECD countries in 2010.

\begin{tabular}{cc}
\hline Country & Percent \\
\hline Australia & $18.6^{*}$ \\
Canada & 14.2 \\
France & 7.3 \\
Germany & 13.2 \\
Italy & 17.8 \\
Spain & $20.1^{*}$ \\
South Korea & 32.1 \\
UK & 8.9 \\
US & 11.8 \\
\hline
\end{tabular}

Note: ${ }^{*}$ Figure in 2009.

cines. However, the principles of government-run health insurance are to provide "basic care" coverage, thus numerous medicines are excluded for various reasons (NRDL in 2009).

The cost for medicines excluded by the NRDL or local government formulary is a key contributor to out-of-pocket medical expenses. Various forms of charity, although still very limited in China, have been a major resource to subsidize patients who cannot afford expensive medications that are not listed for reimbursement by government programs.

Despite the ambitious goal set by the Chinese government and the achievements by government-run healthcare insurance, the financial burden of treating diseases, including drug utilization for catastrophic conditions, remains significant for many people. For example, the average direct medical cost for the treatment of chronic myeloid leukemia (CML), can cost \$5122 USD for patients without bone marrow transplantations and \$50,623 USD for patients with bone marrow transplantations (Table 3); cost on medications is either $2 \%$ or $23 \%$ out of the total medical cost, depending on if marrow transplantations are involved or not (Wang et al., 2009). In contrast, the 2011 annual disposable income per capita was \$3000 USD in China (China annual incomes, 2013). Therefore, without health insurance of any kind or patient assistance programs that often charity organizations and pharmaceuticals would provide, access to innovative medications to treat serious conditions like CML would be challenging for many individual patients and their families in China.

Relatively high expenses on healthcare from individual patients would be part of the potential factors to have significant potential to adversely impact societal development (Whyte, 2010); catastrophic cases on diseases without proper access to healthcare insurance or other reimbursement programs would cause tremendous financial burden to individual patients and their families in China. This issue would also have the potential to generate significant political concern for the government if not appropriately handled, especially when the national economy is rapidly advancing. Therefore, the China government has strived to further reduce the financial burden to both the government system and individual patients by expanding its government run health insurance programs. Among all possible approaches, there is an urgent need to explore additional options to finance the healthcare system, especially to provide affordable access to innovative medications not listed by the national formulary in China.

Discussion on the role of private health insurance has been a topic for health policy for a long time (Private health insurance, 2004; Private voluntary health insurance in development, 2007) with successful examples in developing countries, such as Brazil, where private health insurance has played a critical role in assisting national health care system (Gordon et al., 2013). This manuscript focuses on the concept of using commercial health insurance (CHI) in China as a potential option to assist patients to access medications not listed on the formulary by the government-funded health insurance programs.

\section{Current State of Commercial Health Insurance (CHI) in China}

In China, CHI, under the general scope of the insurance industry, was formally recognized by Chinese government with regulations and related policy in 1998. Currently, the insurance industry in China is regulated under the supervision of China Insurance Regulatory Commission (CIRC) which was also established in late 1998. As supplement insurance coverage to UEBMI, CHI has been focusing on urban areas to provide additional coverage to individuals with the necessary financial resources. Rural areas with excellent economic development have been brought into the focus only recently. However, the rural population remains the minority covered by $\mathrm{CHI}$ in China. In fact, individual disease insurance products were introduced to China in 1995; but it wasn't until 2005 that CHI began specialized operations in China (Chen \& Ye, 2007). A total of 136 million enrollments (nearly $10 \%$ of the total national population) were reported in China in 2002; in Beijing, enrollees of CHI increased from 35,000 to 1 million, a 19\% increase (China insurance regulatory commission, 2010). Although published information is very limited regarding the actual number of people enrolled in CHI programs, the CHI market in China has greatly expanded since 2002 based on the number of companies that currently operate CHI programs and the reported annual premium revenue (Report on health insurance development in China, 2010).

Based on published information by the China government, as of November 2012, there are 61 insurance companies listed by CIRC that are licensed to provide life insurance including health insurance products. These 61 companies are either domestic (36), or foreign-Sino joint ventures (25) depending on the sources of the company's finance. From 1999 to 2012, income on health insurance has been increased by 24 times, from \$596 million USD in 1999 to \$14 billion USD in 2012. The top companies, 
Table 3.

Direct medical costs of treating CML in China (in USD).

\begin{tabular}{ccc}
\hline With Transplantation & Direct Medical Costs & Percentage \\
\hline Transplantation & 33,664 & $66 \%$ \\
Medications & 911 & $2 \%$ \\
Blood products & 11,621 & $23 \%$ \\
Antibiotics and hemopoietic growth factors & 4427 & $9 \%$ \\
Total & 50,623 & $100 \%$ \\
\hline Without Transplantation & Direct Medical Costs & $23 \%$ \\
Medications & 1168 & $53 \%$ \\
Blood products & 2725 & $24 \%$ \\
Antibiotics and hemopoietic growth factors & 1229 & $100 \%$ \\
\hline
\end{tabular}

in terms of amount of annual premiums, include China Life Insurance Company, e-Chinalife, China Pacific Insurance Company, and Ping-An Life Insurance; all are domestic companies. Insurance companies operating in China with overseas funding include Prudential, AIA, Tokyo Marine \& Fire Insurance Company, and Royal Sun Alliance (China insurance regulatory commission, 2013).

As a product line of the China insurance industry, CHI was also created around 1998. In 1999, the premium volume from CHI accounted for $2.6 \%$ out of the total insurance products, by November 2012 this percentage increased to 5\% (Figure 1), reaching a total of \$14 billion USD (China Insurance Regulatory Commission, 2013). The premium volume of all insurance products (excluding health insurance) increased by $11.1 \%$ from 1999 to 2012 while the premium revenue from CHI increased by $24 \%$ during the same period. This trend is an indicator of the significant demands and growth of $\mathrm{CHI}$ in China in the last decade.

In November 2011, CHI programs existed in 36 provinces or large cities in China-a $71 \%$ increase from 2009. As of November 2011, Beijing is leading in terms of annual premium revenue (\$952 million USD), followed by Guangdong (\$93.6 million USD), Jiangsu (\$696 million USD), Shanghai (\$677 million USD), and Shandong (\$651 million USD). Provinces with the least annual premium revenue are located in the western part of China (Tibet and Qinghai, \$10 and \$22 million USD, respectively), and Hainan in the south (\$33 million USD). In 2009 , out of the total national health insurance premium, revenue from medical care treatment products was about $62 \%$; incomes from the other three major products included catastrophic disease (36\%), nursing home (1\%), and loss of insurance due to unemployment (1\%). However, CHI was less than $1 \%$ out of the total national healthcare expenditure in China in 2007 (China Insurance Regulatory Commission, 2010).

CHI accounts for a \$14 billion USD market in China according to annual premiums up to November 2012; however, as shown in Figure 1, this is a very small proportion (around 5\%) of the total insurance premium market in China. This percentage has remained constant over the past several years (Table 4). It is reported that at present, $\mathrm{CHI}$ only covers $10 \%$ of the total medical costs in China (National development and reform commission, China, 2013). If the CHI expenditure is compared to the total health care expenditure, the percentage would become even much smaller, .3\% in 2005, which is the lowest among other Asian-Pacific countries, such as Indonesia, Malaysia, the

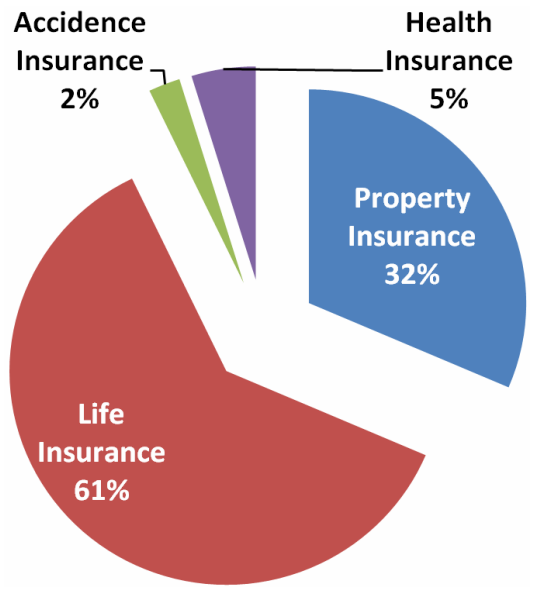

Figure 1.

Insurance Premium Breakdown (\%).

Philippines, Thailand, Vietnam, Papua New Guinea in East Asia; and the Pacific (Drechsler \& Jutting, 2007). A number of factors may contribute to this situation, including a negative perception of having insurance in general, or no encouragement of buying a commercial insurance after the coverage of UEBMI by the government, structure of current $\mathrm{CHI}$ products, and government policy.

\section{Roles that Commercial Health Insurance Could Play to Better Finance Innovative Medicines in China}

There are at least two major roles that $\mathrm{CHI}$ can play in China: 1) to provide medical services and medicines that are not covered by the government plans so CHI works as a supplement to the government programs; 2) to serve as a contracted primary insurer to directly manage the public insurance programs for government agencies with Ministry of Human Resource and Social Security (MHRSS). Both roles are in fact called for by the State Council and explicitly stated in the State Council Health Reform Plan issued on April 6, 2009 (National development and reform commission, China, 2013).

This paper examines the feasibility of establishing a role in China for CHI to provide access to medicines that are not covered by the government plans. In effect, CHI would function as a supplement to government programs. The idea proposed by the authors is that the NRDL would remain the primary target 
Table 4.

Premium Revenue and Payment of Insurance in China from 1999 to $2011^{\ddagger}$ (in 10,000 USD).

\begin{tabular}{ccccc}
\hline & \multicolumn{2}{c}{ All Insurance including Health Insurance } & \multicolumn{2}{c}{ Health Insurance Only } \\
\hline Year & Premium Income & Paid Out & Premium Income & Paid Out \\
\hline 1999 & $2,272,786$ & 832,371 & 59,606 & 17,949 \\
2000 & $2,603,357$ & 860,289 & 106,820 & 21,071 \\
2001 & $3,441,035$ & 975,933 & 100,410 & 54,680 \\
2002 & $4,980,651$ & $1,152,905$ & 199,754 & 81,463 \\
2003 & $6,330,173$ & $1,371,964$ & 394,656 & 114,032 \\
2004 & $7,044,266$ & $1,638,565$ & 145,356 \\
2005 & $8,038,067$ & $1,842,854$ & 423,943 & 176,046 \\
2006 & $9,203,009$ & $2,346,597$ & 204,077 \\
2007 & $11,477,585$ & $3,695,293$ & 614,845 & 190,644 \\
2008 & $15,961,006$ & $4,846,926$ & 285,949 \\
2009 & $18,168,514$ & $5,098,667$ & 95,698 & 354,052 \\
2010 & $23,699,790$ & $5,220,928$ & 955,072 & 430,705 \\
2011 & $23,391,927$ & $6,410,070$ & $1,105,164$ & 586,729 \\
2012 & $25,265,791$ & $7,693,831$ & $1,128,420$ & 486,412 \\
\hline
\end{tabular}

Note: The original data were in RMB and recalculated into USD with the exchange rate of 1 USD = 6.13 RMB.

formulary for (basic) medicines to cover most of the population in China. However, most of the innovative medicines that are not listed by the NRDL would be covered by CHI programs.

Potential benefits of such an alternative would be multifold. First, this would help relieve some of the pressure from the government in the current process of efficiently maintaining and updating the NRDL since the number of medicines, especially innovative medicines, is limited. If more people are covered by $\mathrm{CHI}$ as a supplementary health insurance to the government-run health insurance programs, some of the financial can be transferred away from the government programs. Therefore, the medications that eventually have to be covered by NRDL would decrease as $\mathrm{CHI}$ increases its coverage of this kind. In China, 95\% of the medicines approved in China are generic versions, while the majority of innovative medicines come from outside China. Most of medications covered by the government are generics; overall very few innovative medications are covered. Accordingly, since most of the innovative medications approved by the China FDA are excluded by the public health insurance programs, this has the potential to exert tremendous pressure on the government for access to these medications by the Chinese population.

Second, our proposal that the CHI function to provide access to medical services and medicines that are not covered by the government plans allows $\mathrm{CHI}$ to serve as a supplement to government program. This concept would provide an excellent opportunity for pharmaceutical companies, especially the multinational companies (MNCs), to achieve better strategic planning for market access of these products to patients in China. It would support evaluating the trade-off of being listed by the NRDL versus being covered by CHI. This would also incentivize the pharmaceutical industry, especially the MNCs, to explore a new market segment in China that would help further patient access and generate needed return for further R\&D development in China. Third, the insurance industry (CHI) could also take the advantage of covering more innovative medicines to create a favorable business environment for the $\mathrm{CHI}$ industry to grow in China.

Most importantly, introducing $\mathrm{CHI}$ programs that provide expanded access to healthcare and innovative medicines could actually decrease some of the financial pressure on patients and improve overall affordability, simultaneously decreasing pressure on the government to provide access. With this suggested approach, more healthcare providers and patients in China could be access new and innovative medicines to treat severe medical problems. The recent partnership between Roche and Swiss Re Health Insurance in China to market health insurance to individuals for cancer drugs underscores this point (Roche to boost caner drug sales in China with Swiss Re health insurance partnership, 2012).

\section{Recommendations}

The proposed idea, if implemented properly, would require long-term strategic involvement of myriad industries (e.g., pharmaceutical manufacturers, health insurance, and the China healthcare system) and various perspectives (e.g., business, law/regulations, structure and capacity reconfiguration, etc). We believe our proposal, if implemented effectively, could have significant impacts on healthcare policy in China. Authors of this paper propose five key changes that would need to take place in order to make this proposed idea achieve reality.

Firstly, there is a critical need to recognize and appreciate the value of $\mathrm{CHI}$ as a supplement to government run health insurance programs. Having been operationalized in China for more than 2 decades to supplement to the government programs, $\mathrm{CHI}$ was not widely recognized. Senior government officials have begun to recognize the need to better leverage $\mathrm{CHI}$ as part of the current healthcare reform in China. In many countries, where a comprehensive social health insurance is provided to the population, a high percentage of the population still purchase private health insurance programs (e.g., Canada [65\%], and France [85\%]). In these countries, CHI (known as private health insurance) provides access to services that are not covered by public health insurance. For example, private health insurance programs that are specialized in providing prescription drug benefits are common in Canada since the Canada Health Act, which is the government-run health insurance program, covers healthcare services (such as outpatient visits, hospitalizations), but not prescriptions in outpatient settings. In some markets (e.g., France) private insurance companies offer programs that cover the out-of-pocket costs that are not covered 
by the public insurance system (Wikipedia, 2010).

Promotion of CHI development in China offers a number of benefits, including 1) having more medical treatment/medication options for individuals who are well-off financially and may demand enhanced coverage compared with government programs; 2) helping individuals and families to minimize the risk of catastrophic medical expenses; 3) reducing the financial burden and pressure on the government to provide health care that may not be financially feasible at this point; and 4) when more people, especially the relatively rich seek care using the commercial programs, there would also more room for the relatively poor to obtain care from public facilities through the public programs (Wikipedia, 2010).

Let's take a closer look at the structure of the current CHI products. By examining products listed on their official webpage, it is not too difficult to discover that most health insurance products are run similarly to a term life insurance products. For example, most of the CHI companies offer products called "Insurance for Severe Diseases" that may include cancer treatment. If a person unfortunately suffers from a disease covered by one of these insurance products, a pre-agreed lump-sum payment would be made to the beneficiary regardless of how the payment would be used, and regardless whether this amount would be sufficient enough to cover the expenses incurred to treat the identified medical problem. The human body is so complex and the odds of guessing which part of the body would have a problem for an insurance product in advance is hard to predict. Therefore, it is unlikely that many would have the incentive to purchase such a product, even for those who can afford the premium of the insurance product. In addition, most of the CHI products that are available to the public do not cover medications, especially for outpatient visits. At present, CHI products that do cover both outpatient and inpatient medication expenses, follow the NRDL only. Therefore, the administration cost of running this type of CHI in China would be relatively low; however, these products would not have sufficient features to attract customers who want to buy a CHI product "just in case”.

As more and more insurance companies with foreign funding resources are introduced into China, heavy competition is expected. Foreign companies, such as BCBL and Aetna, who have already had very successful experience running health maintenance organizations (HMOs) in various forms, would introduce their experience and set up health insurance products similar to those found overseas. This is to attract customers, especially those in higher income brackets. A change would be expected in this scenario, that an HMO type of formulary is to reflect the relative higher share of drug expenses compared to other health costs (formulary access, approval process etc.); otherwise, the underlying risk model that in part of any insurance based system would not be successful and would lead to disproportional expenses, putting the entire business model at risk. Insurance systems are all based on the "risk pooling" concept and the assumption that many cover the unlikely event for a few. This requires a balanced risk profile or different premiums for different risk groups especially in the early stage of new insurance scheme.

The second expected significant change would happen to the relationship between these health insurance companies and the healthcare system in China, (i.e., hospitals). Part of the reason that China insurance companies do not have any formulary for drug reimbursement outside NRDL is due to the fact that they have little to no control over what drugs are prescribed for which conditions. The current incentive system may lead to a potential undesired prescribing behavior. Therefore, guidelines to encourage physicians and hospitals to follow HMO formulary recommendations would be an important task.

The third significant change is expected to happen to the pharmaceutical industry, both domestic and foreign. All MNCs in China and most of the top domestic pharmaceutical companies in China have departments submitting proposals to the government agencies either for pricing considerations or for listing in the NRDL considerations. Creating a successful partnership between MNCs and the CHI not only requires an understanding of how to work with HMO-type of CHI, but it is also dependent upon applying good evidence-based data in determining which medications are to be included in the $\mathrm{CHI}$ formulary. Therefore, some fundamental new activities would be needed, such as a new scope of agreements or contracts between a pharmaceutical company and a CHI company in China (i.e., business capacity in terms of planning and pricing negotiation) and medical team capacity providing necessary clinical supports. Other considerations would include the process for establishing a formulary; a consensus on the criteria used to evaluate innovative medicines for inclusion in a CHI formulary; who are the key stakeholders that should participate in the creation and maintenance of a formulary; and how to handle conflict among CHI, the pharmaceutical companies, and the healthcare system on inclusion or exclusions of treatment options. Since all of these issues have been addressed previously in other markets, it is possible to determine a comprehensive approach; but it would certainly take time and great efforts to make it happen. There would be important items or areas that would require close cooperation between the $\mathrm{CHI}$ industry, the pharmaceutical industry, the academic researchers, and the government.

The fourth change that needs to take place is to create a legal framework that would provide good incentive to people to buy CHI. Many examples can be gleaned from countries where commercial health insurance has been well established while the public health insurance is the dominate force in providing finance to health care. For example, in Australia, all citizens are covered by the government-run universal health insurance. Yet, depending on age groups, the amount covered can be 30\%, 35\%, and $40 \%$, the Australian government subsidizes premiums of all private health insurance coverage for both hospital admissions and services at ancillary setting. On the other hand, if a person does not join a private health insurance plan after reaching 31 years of age, an annual premium must include a loading of $2 \%$ per year if the person goes without hospital coverage after this critical date (Wikipedia, 2010); therefore, setting up proper law or regulations to encourage participation in $\mathrm{CHI}$ is critical.

The fifth significant change would be to enhance the accuracy of the data, both clinical and accounting information at a patient level, from hospitals in China. This data would be shared with CHI companies. For a successful CHI, it is critically important to perform a good actuary to estimate an appropriate premium for providing medical insurance products to patients for medical care including covering new innovative drugs that are not included in the government reimbursement list. This would also be the way to properly evaluate possible risks in providing such coverage in this kind by $\mathrm{CHI}$ programs in China. 


\section{Summary}

Healthcare reform in China is in a very dynamic and exciting phase, leading to great improvements in access to health care for the people in China. Numerous changes are expected and there is a great opportunity for all stakeholders to be involved, including further growth in the $\mathrm{CHI}$ and pharmaceutical industry as well. A critical issue is how to properly fund medicines that are not covered by the NRDL. This paper explores CHI as a viable option to finance innovative medicines that are not listed by the NRDL. To make this proposal successful in reality, more work and further research will be required. However, once the concept/idea is well accepted among all stakeholders and then implemented, optimal benefits will be significant for patients, the Chinese government and the healthcare industry by contributing to the economic development and further improvement in health outcomes in China over the next few decades.

\section{Acknowledgements}

Authors of this manuscript are grateful to Julliana Newman, ELS, for her professional editing on this manuscript.

\section{REFERENCES}

Bhattacharjye, A. S., \& Sapra, P. K. (2008). Health insurance in China and India: Segmented roles for public and private financing. Health Affairs, 27, 1005-1015. doi:10.1377/hlthaff.27.4.1005

Chen, T., \& Ye, X. (2007). Private health insurance and health actuary in mainland China. Proceedings of the 14th East Asian Actuarial Conference, Tokyo.

China annual incomes (2013).

http://money.cnn.com/2012/06/26/news/economy/china-middle-class /index.htm

China government announcement (2012).

http://www.google.com/search?q=ziyuanzhongxin+shujuziliao+book $335927+$ site:www.chira.org.cn

China healthcare reform (2012)

http://www.chira.org.cn/xinwenzhongxin/jiaodianxinwen/new33779 7.html

China Insurance Regulatory Commission (2013).

http://www.circ.gov.cn/web/site0/tab61/

China Insurance Regulatory Commission (2010).

http://www.circ.gov.cn/web/site0/tab211/i47326.htm

China Insurance Regulatory Commission (2013). http://www.circ.gov.cn/web/site0/tab454/i191553.htm

Drechsler, D., \& Jutting, J. (2007). Different countries, different needs: The role of private health insurance in developing countries. Journal of Health Politics, Policy and Law, 32, 497-534. doi:10.1215/03616878-2007-012

Gordon, R., Xu, T., \& Xie, L. (2012). Brazil's health insurance. http://www.google.com/search?safe=active\&rlz=1T4MXGB_enUS5 46\&q=Ronald+Gordon+and+Tian+Xu\&oq=Ronald+Gordon+and $+\mathrm{Ti}$ an+Xu\&gs_l=serp.12..0i22i30.24027.62191.0.63861.56.40.0.0.0.0.3 339.10282.0j1j4j2j6-1j0j1j2.11.0....0...1c.1.22.serp..46.10.10048.q8u uFXd4Z8s

Hougaard, J. L., Østerdal, L. P., \& Yu, Y. (2008). The Chinese health care system: Structure, problems and challenges. No. 08-01. Discussion papers department of Economics University of Copenhagen, Copenhagen.

Le Deu, F., Parekh, R., Zhang, F. N., \& Zhou, G. (2012). Healthcare in China: "Entering uncharted waters". New York: McKinsey \& Company.

MOH China statistics (2012). http://www.moh.gov.cn/publicfiles///business/cmsresources/mohwsb wstjxxzx/cmsrsdocument/doc15055.pdf

National development and reform commission, China. (2013). http://shs.ndrc.gov.cn/ygid/ygwj/t20090408_271138.htm

NRDL in 2009 (Chinese medicines listing) (2013). http://www.china.com.cn/news/txt/2009-08/19/content_18359838.ht $\mathrm{m}$

OECD (2011). Health: Key table from OECD.

Private health insurance: Implications for developing countries. Discussion paper (2004). World Health Organization, Geneva.

Private Voluntary Health Insurance in Development (2007). World Bank, Washington DC.

Report on Health Insurance Development in China (2010). Beijing: China Insurance Regulatory Commission.

Roche to boost cancer drug sales in China with Swiss Re health insurance partnership. HIS. Global Insight (2012).

Sussmuth-Dyckerhoff, C., \& Wang, J. (2010). China health care reforms. McKinsey. Health International.

Wang, J. X. et al. (2009). Overview of chronic myelogenous leukemia and its current diagnosis and treatment patterns in 15 hospitals in China. Chinese Journal of Hematology, 30, 721-725.

Whyte, M. K. (2010). Fair versus unfair: How do Chinese citizens view current inequalities? Growing pains: Tensions and opportunity in China's transformation.

Wikipedia (2010) Health insurance. http://en.wikipedia.org/wiki/Health_insurance 